\title{
The effects of vaccination in case of influenza on the elderly in Hong Kong in reducing flu infection
}

\author{
S M Nazmuz Sakib \\ Graduate of BSc in Business Studies \\ School of Business And Trade \\ Pilatusstrasse 6003, 6003 Luzern, Switzerland \\ sakibpedia@gmail.com \\ Student of Department of Law \\ Dhaka International University \\ House \# 4, Road \# 1, Block - F, Dhaka 1213 \\ sakibpedia@students.diu.ac
}

\begin{abstract}
The reason for this dissertation is to establish the effects of vaccination on the elderly (>65 years old) in Hong Kong in reducing flu infection. Influenza vaccine uptake in the elderly (>65 years old) in Hong Kong significantly increased in 2003 after the SARS epidemic. The exact impacts of influenza vaccine among the elderly in Hong Kong are a subject of contention. The effectiveness of the influenza vaccine comes from observed studies which may be prejudiced since it is difficult to identify and justify the evidence. A review of various literatures has shown that influenza causes serious illness and death particularly highly vulnerable groups such as adults aged 65 years and above. Therefore, more efforts should be initiated to reduce mortality caused by influenza among the elderly. According to the WHO (2005), vaccination is
\end{abstract}


among the most effective approach for preventing death associated with influenza to vulnerable groups such as the elderly.

Keywords: influenza vaccine, influenza, vaccine, epidemiology, vaccine effectiveness, and perceived barriers.

\section{Literature Review}

\section{History of influenza}

Influenza virus has been in existence and spreading around the world since the $16^{\text {th }}$ century, its pathogens causing global pandemics and epidemics. According to Horimoto \& Kawaoka (2005), different pandemics have appeared in the $20^{\text {th }}$ century: Spanish flu (H1N1) that occurred in 1918, Asian flu (H2N2) of 1957, Hong Kong flu (H3N2) in 1968, Russian flu (H1N1) in 1977 and H1N1 in 2009. The Spanish flu that occurred in 1918 was the most fatal after causing more than 50 million deaths globally. The Asian flu (H2N2) of 1957 led to more than 1 million deaths, the Hong Kong flu (H3N2) in 1968 resulted in 700,000 deaths. The most current influenza Russian flu (H1N1) in 1977 and H1N1 generated approximately 17,000 deaths (Mullooly et al. 1994).

\section{Viral Etiology of Influenza Virus}

Influenza viruses are categorized under Orthomyxoviruses which is the larger family of Ribonucleic acid (RNA) viruses. Noda \& Kawaoka (2010) defines the influenza virus as an eight-segment and single-stranded RNA genome which encodes 10 viral protein and surface molecules like the neuraminidase $(\mathrm{N})$ and haemagglutinin $(\mathrm{H})$. There are three categories of influenza viruses: Type A, Type B, and Type C. type A virus causes infection in mammals, 
birds, horses, swine, and so forth, and it presents a risk to human beings. Type A influenza virus has been linked to various pandemics and the highest morbidity and mortality rates. Both Type B and Type $\mathrm{C}$ viruses cause infections in people only. Type B influenza is the same as Type influenza. According to clinical presentation Type, A influenza usually affects young adults and children. Influenza Type $\mathrm{C}$ is modest and does not result in infections or epidemics but can cause mild respiratory infections in both adults and children.

Type A influenza causes a lot of pandemics and comprises haemagglutinin (HA) (H1H16), 16 glycoproteins, and 9 neuraminidase (NA) (N1- N9) subtypes that were extracted from birds, sea mammals, horses, pigs and humans (Horimoto \& Kawaoka 2005). Three subdivisions of HA type (H1, H2, and $\mathrm{H} 3$ ) have been found among humans. Influenza B generally happens between two to four years. Type $\mathrm{C}$ Influenza is usually related to periodic and subclinical infections. The 1918 Spanish influenza was caused by the first subdivision of the H1N1 virus. The next subdivision H2N2 was responsible for the 1957 Asian influenza comprised of PBI (polymerase basic 1), HA (H2), and NA (N2). The third subdivision H3N2; H3N2 which has HA (H3) and PBI division in the background of the human gene caused the 1968 Hong influenza.

\section{Modes of Transmission}

Understanding the mode of influenza transmission and the period of infections are vital factors of control measures such as vaccination. Influenza virus reproduces in the epithelium of the respiratory tract; penetrates the body through the nose and mouth. Infected hosts can discharge virus into the air during sneezing, coughing, talking and breathing producing particles which are of different sizes. Three paths of transmission well known include droplet which is large particles and can fall on the upper respiratory tract but due to the size, they cannot be transferred into the lungs (Zhang et al. 2018). Aerosol and small particles that can remain 
hanging in the air for longer duration and they can land into the lower respiratory tract. This droplets nuclei causes severe illness. Contact transfer involves the transfer of contagious particles to other membranes through direct contact of contaminated objects. Sometimes it can be transferred through indirect contact of contaminated objects. More research should be conducted to completely explicate the epidemiology of the spread of certain ARIs from one elderly to another especially those in care homes in Hong Kong (Wong et al. 2004).

\section{Droplet and aerosol transmission}

The significance of these routes of influenza spread is not well known and the involvement of aerosolized contagious droplets of nuclei has been a debate of contention. Droplets with the diameter of $<8 \mu \mathrm{m}$ comprise more than $99 \%$ of the spray during cough; therefore, coughing can produce a lot of small particles. However, their volume is low and most of the virus particles are infected in larger droplets that do not scatter extensively hence proximity is needed for transmission to occur. Although studies done by Gupta, Y.K. \& Padhy, (2010) established that influenza viruses can survive artificial airborne aerosols for some time, the importance of their studies about the natural routes of influenza infection has been doubted by other researchers. Disease transmission through aerosols is affected by host factors such as viral load, site infection, respiratory activity, and incidences such as particle aggregation, evaporation, and relative humidity. However, the direct confirmation that naturally developed aerosolized particles can endure the journey and reach the susceptible hosts and transmit disease is wanting. More research on the transmission of influenza virus is required. An aerosolgenerating process is described as a medical process that can stimulate the production of aerosols into different sizes such as droplet nuclei. 
Studies on the epidemiology of influenza transmission have overlooked to address the spread of the virus from one elderly to young health care workers during the aerosol-generating process, especially in regards to other pathogens. This gap arises because different kinds of literature fail to fully define the aerosol-generating process. Additionally, there is insufficient information concerning the minimum ventilation requirement to minimize pathogen spread during these processes. There is no confirmation to indicate a difference in the efficacy of particulate respirator on face masks as a constituent PPE for regular care. However, further research is required to establish if there is a difference between medical masks and the efficacy of particulate respirators in regards to the aerosol-generating process that has been constantly linked to the risk of pathogen transmission. The different medical intervention involves in a risk of generating aerosols, which has outcomes for the recommendations for infection control and personal protection measures. Kwong et al (2010) performed a methodical evaluation of 10 nonrandomized types of research that appraised the risk of transmission of severe infections to elderly in a hospital set up in Hong Kong undergoing aerosol-generating processes compared to those not undergoing the process. The study found a considerably increased risk of transmitting an acute respiratory syndrome among those that did receive the influenza vaccine.

\section{Influenza Symptoms}

Influenza (flu) is explained as an infectious, respiratory sickness caused by influenza viruses. It is responsible for the upper and lower respiratory tract infections which include throat, nose, and lungs. At times, such infections can be mild, extreme, and even result in the death of the patients. Signs and symptoms of influenza consist of cough, running nose, headaches, body aches, chills, muscle aches, fever, stuffy nose, fatigue, and feeling nauseous. Diarrhea and vomiting are more prevalent among children (CDC 2013). 


\section{Influenza Vaccination}

\section{Vaccine types}

Influenza vaccination is considered to be the most effective way of preventing influenza, complications associated with the flu and helps to reduce influenza-associated hospitalization and mortality. The current registered Seasonal Influenza Vaccines (SIVs) in Hong Kong comprises live attenuated influenza vaccine (LAIV) and an inactivated influenza vaccine (IIVs). Two different types of influenza B (Victoria and Yamataga lineages) are found all over the world. Trivalent SIV includes three types of seasonal influenza viruses: one influenza A (H3N2) virus, one influenza A (H1N1)pdm09 virus, and one influenza B virus (either a Yamagata or Victoria lineage). Quadrivalent SIV comprises an extra influenza B virus of lineage which is not found in the trivalent SIV. Investigations on Quadrivalent SIV demonstrated that the inclusion of an extra influenza B strain failed to lead to immune interference to different strain added in the vaccine. Additionally, the levels of undesirable occurrences following Quadrivalent SIVs and trivalent were the same. It is anticipated that Quadrivalent SIV might offer extra protection against influenza B, particularly in seasons largely supporting the influenza virus. SIV is recommended to be administered annually. A good number of inactivated influenza vaccines (IIVs) are administered through intramuscular route and are required to be used in persons above six years apart from those with contraindications. The only one type that is available locally for use in persons aged two years and must be administered via intranasal is known as LAIV (Flumist).

After the introduction of the first influenza vaccine in the 1940 s, vaccinations have contributed to the protection of the public from the influenza virus. Reed et al (2014) documented that influenza vaccination has resulted in 90,000 less hospitalization and few sicknesses. 
Based on the research conducted by Barr and Jelley (2012), influenza vaccines are different among diverse groups of people because they are recommended for administration to different types of vulnerable and healthy people. To achieve efficacy, the influenza vaccine should be administered annually since the virus strain transform with each season. Influenza vaccinations are significant in maintaining a good percentage of the people immune to infectious diseases and avoid further transmission of the disease, this phenomenon is widely referred to as herd immunity or community immunity. Every year, the influenza vaccine comprises the strains of both the influenza A and B viruses which investigators project will be overriding for the influenza period. When the vaccine is equivalent to the influenza virus that is spreading, then it has strong efficacy to protect the population from influenza Soema et al. (2015). The most common Influenza vaccines are categorized as live attenuated influenza vaccine (LAIV) and inactive influenza vaccine (IIV).

Influenza vaccines are additionally classified as trivalent and quadrivalent vaccines. Trivalent vaccines consist of two influenza A and a single influenza B strains. The production of IIVs is done via influenza viruses developed in chicken eggs and later made inactivated (Hamborsky \& Kroger 2015). The administration of IIVs is done by needle injection to the skin or muscle creating an immune response. The body produces antibodies to shield against influenza virus strains which are incorporated in the vaccine. However, for patients who are allergic to an egg are given another option known as the recombinant vaccines. LAIVs are manufactured through egg-based production procedures; however, the virus is weakened rather than inactivated. Such vaccines are administered via nostrils and are referred to as nasal sprays. There are no predilections in the use of both IIV and LAIV, since all of them offer immunity 
against influenza. Though, LAIVs are generally produced to be used by people between 2 years to 49 years.

\section{Influenza Vaccination among the Hong Kong Elderly People (age above 65)}

Zhang et al. (2018) documented that the chances of elderly people in Hong Kong (>65 years) to die from influenza is 14 times compared to midlife and young adults. Based on the 1996-2012 statistics of annual hospitalization counts in Hong Kong, influenza was discovered to be highly related to hospital admissions for acute respiratory illnesses, with noticeable and large rates being elderly above 65 years. Hong Kong's seasonal influenza is illustrated by a winter peak approximately the first quarter of every year and the third quarter. Following the main summer influenza season prevailed by A (H3N2) in 2019, was low in September and November. Just like other states and regions in the temperature region of the Northern Hemisphere, seasonal influenza begun to increase in late December 2019, become high in January and February and reduced to baseline level in June 2020. Based on the influenza virus subtyping performed by the Public Health Laboratory Service Branch (PHLSB), influenza viruses categorized from the $44^{\text {th }}$ week of 2019 to the $13^{\text {th }}$ week of 2020 were influenza B, and $90 \%$ of them were from Yamagata family. The entire 2019 winter season was dominated by influenza B/Yamagata.

During the peak influenza season, the elderly are more susceptible to influenza. Therefore, elderly people are considered to be a risk population because the population is generally aging and will increase extremely in the coming years. This results in the prevention measures to contain the disease and the government has given this population a priority. However, it is a costly measure for health care facilities to offer treatment to this population which makes the government adopts prevention. The government advised people to observe key prevention measures like hand washing, wearing face masks, using antibacterial sanitizers, and 
above all vaccination. The Hong Kong government started the Residential Care Home Vaccination Program in 1998 to offer free IV for people of the Resident Care Homes for the Elderly (RCHE). Currently, elders above 65 years with severe medical conditions, holders of valid Certificate for Waiver of Medical Charges, and those getting Comprehensive Social Security Assistance (CSSA) are entitled to free seasonal IVs sponsored by the Government Vaccination Program. The government established the Elderly Vaccination Subsidy Scheme in 2009 , to promote all elderly above 65 years who receive pneumococcal and influenza vaccination from private health practitioners. The subsidy amount for the seasonal influenza vaccine amounts to $\$ 210$ for each dose.

\section{Efficacy and effectiveness of influenza vaccines}

Vaccination is among the most effective public health tools presently available to protect people from influenza infections. Vaccine effectiveness (VE) is determined by the relationship between virus strains available in the vaccine and the virus spreading in a particular community. Some studies have indicated that when strains in IV tend to match with those circulating, vaccinated people are $60 \%$ less likely to get the flu compared to those who are not vaccinated. As demonstrated by the World Health Organization (WHO), if the strain of the vaccine directly matches the spreading influenza viruses, efficiency of IIVs in people with less than 65 years usually vary from $70 \%$ to $90 \%$, while the effectiveness of IIVs in preventing influenza infection in the elderly above 65 years is at best modest, despite study design, population, and setting. Darvishian et al. (2017) studied meta-analysis and established that the effectiveness of influenza vaccination of SIV against influenza among the elderly in the community during the influenza period was $44 \%$ in matched seasons and only $20 \%$ in mismatched periods. The same was found by other studies of Rondy et al. (2017) which grouped 
SIV and VE against influenza-related hospital admissions which accounted for 37\% among the elderly aged 65 and above.

Early estimates of influenza vaccine efficacy by Wong et al (2004) suggested that influenza vaccines were $80 \%$ effective in reducing flue infection among the elderly. However, the real effectiveness of the influenza vaccine has been discussed by different researchers. Two meta-analyses study by Leung (2007) revealed much lower efficiency than initially believed. All meta-analyses showed that administering the influenza vaccine in the elderly such as the MF59adjuvanted influenza vaccine was effective for averting hospitalization because of influenza or pneumonia-related complications although its effectiveness ranged from 40 to $50 \%$. One metaanalysis comprised only community elderly persons in Hong Kong, while the other also incorporated institutionalized elderly persons. The outcomes of the previous reviews indicated that the effectiveness of hospitalization due to influenza was higher in the institutionalized elderly 36 to $47 \%$ compared to community based elderly $25-33 \%$. Although the meta-analyses and other studies showed significant effectiveness for preventing hospitalization associated with influenza, most research findings included other severe acute respiratory infections (SARI) and failed to concentrate on influenza only. Additionally, most studies were undertaken when in the previous years when influenza vaccine intake was still very low and antiviral agents were limited. In the current context, which has high rates of influenza vaccine uptake, the herd effect can be very significant. This means that early treatments and vaccinations can reduce the possibility of influenza infection among the elderly in Hong Kong. More studies are needed to understand clearly how the influenza vaccine is important to the elderly in the modern context, described by high vaccine intake. 
Jefferson et al. (2005) found out that only the inactivated vaccine is approved to be used in adults 65 years and above. The most current research of Liu (2010) suggested that in the elderly population, vaccines were not the most appropriate solution to reduce influenza and were slightly effective in elderly found in long term care institutions and nursing homes. Constant with a low level of effectiveness in reducing influenza, the vaccines have been discovered to induce weak antibody responses in the aged population. Vaccine effectiveness has been found to be low among the elderly who are at sensitive threat for post-influenza pneumonia. Vaccines fail to achieve their objectives during periods with an antigenic mismatch. Even though the elderly in Hong Kong are recommended to undergo yearly influenza vaccination, there are other issues such as blunted antibody response following frequent vaccination.

As demonstrated in Chan et al. (2019) study, the vaccine effectiveness (VE) indicated that the influenza vaccine provided moderate protection to the elderly population from cases of hospitalization. Additionally, few elderly people that had received the vaccines during the 2017/2018 winter season were affected by influenza A (H1) and influenza B. The study approximations showed that influenza vaccination provided better protection against confirmed cases of influenza cases in Hong Kong during the 2017/18 winter season. In the study based on an RTC, Verhees et al. (2019) could not show any connection between influenza vaccination and death or particular causes of mortality in the elderly after a follow up of 20 years. Additionally, the authors did not find any effect of vaccination as previously reported by other researchers. Despite not being statistically important, survival favored the vaccine group after the whole period of follow-up, with risky levels being constant during that time. The analysis of subgroups showed that the effects of influenza vaccination on deaths could be more common among elders below 64 years Verhees et al. (2019). This fact is supported by other studies which stated that 
clinical effectiveness of the vaccine and influenza humoral immunity is disapprovingly contributed by age. Therefore, it is significant to anise the young elderly on the contribution of age to influenza vaccination. Additionally, influenza-related hospitalization and deaths were also substantial in the young elderly $<65$ year.

Verhees et al. (2019) analyzed other subgroups based on pre-existing pulmonary and cardiovascular diseases on the earlier vaccination which did not demonstrate clear suggestions for the effects of the vaccine as the cause of mortality. The same was found in other studies of Sung et al. (2014) which established that although the elderly in Hong Kong with lung diseases could be more vulnerable to harmful effects of influenza, there was no clear evidence that influenza vaccine had effects on pulmonary-linked mortality. Scholarly, it is complicated to make a comparison of the study results with other literature because different writers mainly assessed the seasonal effects of influenza vaccination for a short period (Verhees et al. 2019). For example other research RCT, Praditsuwanet al. (2005) only evaluated mortality in one year. While underpowered for the secondary outcomes, the authors could not show the correlation between influenza vaccines and mortality among the elderly. A cohort study indicated that influenza vaccination among the elderly reduced mortality during the six months follow-up, but failed to avert death in healthy elderly. Nevertheless, in the investigation vaccination reduced deaths among the elderly with comorbidity (Verhees et al. 2019). Additionally, a meta-analysis assessing the effects of influenza vaccine among the elderly revealed that IV could reduce mixed cardiovascular mortality.

According to Kavian et al. (2020), the high-dose influenza vaccine is more efficient compared to the standard dose among the elderly ages 65 and above. The study was conducted to see whether a high dose vaccine was effective in protecting the elderly compare to normal 
vaccines. The authors concluded that high-dose vaccine is safer, stimulates considerably higher antibody responses, and offers greater protection against influenza sickness compared to normal dose in people older than 65 . Their study data also showed that the high-dose vaccine could provide clinical gains for preventing hospitalization, cardio-respiratory conditions, pneumonia non-routine medical use, and medical visits. The researchers documented the Fluzone High-Dose vaccine as the inactivated influenza vaccine that comprises four times the amount of antigen found in the standard-dose Fluzone vaccine. They also established that the High-Dose vaccine is a drug manufactured particularly to address the age-associated reduction of immune systems among older adults.

Similar findings by Girard et al. (2013) also suggests that three new vaccines, comprising immune-boosting adjuvant and higher doses of antigen produced greater immune response compared to the normal edition of the influenza vaccine. The research was performed in Hong Kong and is aimed at elderly people aged 65 and over to offer extra boost due to their reduced immunity. For the research, 1861 adults from ages 65 to 82 were haphazardly given the ordinary quadrivalent flu vaccine or the newer vaccines manufactured for seniors including the trivalent MF59-adjuvanted vaccine (FluAD), (FluZone) and the high-dose trivalent (three-strain) vaccine. The participants received their immunization from November 2017 to February 2018 and also got vaccines strains for the Northern Hemisphere flu season that occurred in 207-18. The investigation established that all the three new senior formulations elicited a $b$ greater immune response founded on antibody level compared to the H3N2 and H1N1 flu strains. The subjects who received the normal vaccine had a 3.4 fold antibody rise to the $\mathrm{H} 3 \mathrm{~N} 2$ vaccine element than with a 4.2-4.7 fold increase among those who got the improved vaccines. The investigators witnessed one major difference in the three improved vaccines. Elderly people who got the 
recombinant high-dose vaccines witnessed better antibody response to the H3N2 cell-like virus that related to the H3N2 strain which was spreading in the community. Reaction to the influenza B Victoria strain was the same in all the three new vaccines, although antibodies were slightly higher in participants who received the high-dose vaccine. The writers were, although careful to conclude that immune response did not warranty clinical protection. However, their research has potential and presents a greater groundwork for further effective trials which may shed more light on different clinical benefits of the new products. Although other current studies are constant with personal studies that dented the newly improved flu vaccine against the previous version, more research should be conducted to unswervingly compare the ability of each vaccine to prevent serious illness.

As shown by Fukushima and Hirota (2017) in their study, VE presented reasonable fine protection against influenza among the elderly in the influenza B/Yamataga 2018/2019 winter period in Hong Kong. Their estimates indicated that the 2019 SIV was efficient against both influenza A (H1) and influenza B infections. Because of fewer cases of influenza A (H3) discovered during the research period, the VE estimation for such kind of subtype was statistically insignificant due to power (Fukushima \& Hirota 2017). The general VE estimation of $60 \%$ for all influenza is related to high season approximation in Canada $55 \%$ and $54 \%$ in Europe but slightly more than the United States 42\%. Hong Kong used higher QIV with influenza Yamagata than other regions. The writers noted that there was a co-circulation of influenza A (H1) with Hong Kong's influenza B/Yamagata while there was a co-circulation of influenza A(H3) with Europe's' influenza B/Yamagata. The study estimates revealed that SIV presented better protection of influenza infection in the 2019 winter season among the elderly in Hong Kong. 
During the winter influenza period, most of the people clinically at a greater risk of contracting a severe influenza illness are those aged 65 years and above. A study conducted by Zhang et al. (2019) discovered that only $47.1 \%$ of elderly people in Resident Care Homes for the Elderly (RCHE) received the vaccination, but the number increased to $50.2 \%$ during the winter. This is due to the fact that elderly people were likely to get infected with the influenza virus during the period. The WHO has set up a goal of ensuring that $75 \%$ of people who are 65 years and above must get the influenza vaccination during each winter period. The WHO and the government of Hong Kong have been trying to increase the general uptake of the influenza vaccination. The government of Hong Kong established an upshot national publicity program. More than $72.8 \%$ of individuals with 65 years and above were subjected to influenza vaccination during the 2017/2018-winter season (World Health Organization 2015).

The influenza vaccination is believed to be an important clinical procedure that could considerably decrease the number of elderly persons in developing the influenza infection and presenting a meek effect in minimizing the complications and death that can emanate from the influenza virus. Further, the study by Chan et al. (2019) proved that influenza vaccination reduced cases of hospitalization and particularly in older people staying in the community. However, the researchers discovered that influenza vaccination was less efficient and the level of protection was short-lived in elderly individuals, in comparison with healthy living adults. The effectiveness of the vaccination for elderly individuals was $50-70 \%$ during their stay in institutions. The rates were fewer efficacies due to the lower immunogenicity present in the elderly individuals but the results also discovered to have $60 \%$ effectiveness in decreasing influenza illness and also can prevent the rates of hospitalization and deaths up to $80 \%$. Influenza has also shown to minimize the likelihood of increasing additional problems like pneumonia and 
bronchopneumonia (Chan et al. 2019). Equally, there are different apprehensions that the effectiveness of the influenza vaccination in such studies has been overrated.

\section{Adverse Effects}

Adverse effects on the elderly after the administration of IIV include pain, swelling, and redness of the injected part. Other systematic symptoms like chills, fever, myalgia, and malaise only affect $1 \%$ of the recipients. Other different adverse effects can include Guillain-Barré syndrome (GBS) and anaphylaxis. GBS is defined as a severe paralyzing disease, generally aggravated by earlier contamination, surgery, or sometimes after immunization. GBS is illustrated by progressive weakening of limbs and areflexia. Most current researches have assessed the risk of GBS among the elderly 65 years and above. Babazadeh et al. (2019) found that there was no strong relationship between cases of influenza vaccination and GBS. Their study evaluated a few cases of GBS and found out that an increase in the cases of GBS was not likely to happen to the elderly who had received influenza vaccination. The study further revealed that no scientific has associated GBS and influenza vaccine, and most commonly occurs in incidences of pandemic vaccines. They argued that there should be no ban on the administration of the influenza vaccine on the elderly, except when an individual has a history of GBS for a period of six weeks after vaccination. Additional findings by Goodfellow and Willison (2016) related to GBS incidences and seasonal winter patterns in Hong Kong. A cohort study showed that influenza vaccination among the elderly reduced mortality during the six months follow-up, but failed to avoid death in healthy elderly. However, in the investigation, vaccination reduced deaths among the elderly. A study that checked the seasonal model of GBS in 2009 documented that an increase in influenza vaccination coverage from $19 \%$ to $35 \%$ did not result in any single case of GBS. These findings show a significant relationship between 
hospitalization because of influenza and GBS case, but the influenza vaccine did not have any effects on the GBS pattern. In the case of LAIV, the most prevalent effects after LAIV is administered include running nose of the elderly. It is more serious to asthmatic elderly which can increase whizzing.

\section{Perceived Barriers of Influenza Vaccination}

In the recent past, numerous investigators have emphasized on discovering possible obstacles to influenza vaccine acceptance among the elderly. Primarily Zhang et al. (2018) described the notion of vaccine hesitancy. Vaccine hesitancy is used to define the reception of different vaccines on a variety amid demand and lack of demand varying from agrees to all vaccines to allowing no vaccine. According to Zhang et al. (2018), vaccine hesitancy means the delay in getting or rejection of vaccination regardless of the availability of vaccination services. Vaccine hesitancy is difficult and perspective specific, differing across vaccines, place, and time. Based on this definition of vaccine hesitancy, it proposed that barriers to vaccine acceptance can be different in significance and kind, concerning the disease and vaccine in the picture. An influenza vaccine, when compared to other ordinary vaccines, presents unique distinctiveness that must also be measured when focusing on influenza vaccine uncertainty. This includes vaccine effectiveness varies yearly and is normally low.

Influenza vaccination is a requisite every year in countries like Hong Kong, it is recommended for the elderly above 65 years and there are several influenza myths such as the flu shot may result in flu. Therefore, influenza vaccine hesitancy is a term that has exceptional features that substantiate more studies to gain a particular considerate of the occurrence (Zhang et al. 2018). Earlier methodical reviews on forecasters of flu vaccine uptake in the elderly give primary imminent to appreciate influenza vaccine hesitancy in the elderly. It is vital to recognize 
the issues forecasting IV behaviors amongst the elderly since such factors can offer a guideline to the plan of intervention measures to enhance IV rates. Various factors such as lack of important advice from health care providers, lack of health insurance, perceived good health, and health risk factors, negative perception on vaccine safety and efficacy, and panic over side effects have been discovered to be affecting influenza vaccine uptake among elderly people in Hong Kong. Previous researchers also documented that the elderly, apparent side effects, apparent effectiveness, advice from health caregivers, and accessibility of vaccines were definitely associated with IV uptake among the elderly in Hong Kong (Zhang et al. 2018).

\section{Literature on Methodology}

A review of the present study on the effects of vaccination on the elderly ( $>65$ years old) in Hong Kong in reducing flu infection established that most studies were based on two main types of studies that determine the effectiveness of influenza vaccines on the elderly. The kinds of literature used observational studies and randomized controlled trials.

\section{Observational studies}

Most studies used different types of observational studies such as case-control and cohort studies. The pieces of literature evaluated how influenza vaccines work among the elderly by drawing a comparison between those who had been vaccinated against those not vaccinated. Vaccine effectiveness is described as the reduction level in the occurrence of influenza illness in vaccinated people in comparison to those that are not vaccinated, typically with alteration factors that are related to influenza sickness and vaccination. The adjustment factors include the existence of severe medical conditions. In the meta-analysis, influenza vaccination rates were above average. The studies indicated that influenza vaccination was effective among the elderly. 
A good number of observational studies showed an increase in influenza vaccine effectiveness in elderly people with primary health conditions.

\section{Randomized controlled trials (RCTs)}

This kind of study design is where participants are assigned arbitrarily to get the influenza vaccine. Most works of literature measured the vaccine efficacy by drawing comparisons between the influenza illness in vaccinated and unvaccinated groups. Most investigators preferred RCTs because it helps to reduce prejudice which could result in invalid results. Most of the current literature documented applied reviews of theories, quantitative and cross-sectional research designs, have concentrated on different predictions and explanations to search for or accept health interventions such as vaccination to make informed health choices.

\section{What is known in the literature?}

It is believed that the influenza virus is the major cause of mortality and morbidity. Alders are considered people at higher risk because the disease presents serious complications that lead to hospitalization and even death. During the peak influenza season, the elderly are more susceptible to influenza. Therefore, elderly people are considered to be a risky population because the population is generally aging and will increase extremely in the coming years. This results in the prevention measures to contain the disease and the government has given this population a priority. According to the WHO, vaccination is among the most effective approach for preventing death associated with influenza to vulnerable groups such as the elderly. The government of Hong Kong established the Elderly Vaccination Subsidy Scheme in 2009, to promote all elderly above 65 years who receive pneumococcal and influenza vaccination from private health practitioners. Administering influenza vaccine in the elderly such as the MF59adjuvanted influenza vaccine is effective for averting hospitalization because of influenza or 
pneumonia-related complications although its effectiveness ranged from 40 to $50 \%$. The elderly population, vaccines are the most appropriate solution to reduce influenza and are slightly effective in the elderly found in long term care institutions and nursing homes. The literature has potential and offers a greater groundwork for further effective trials which may shed more light on different clinical benefits of the influenza vaccination and new vaccines. Although other current studies are constant with personal studies that dented the newly improved flu vaccine against the previous version, more research should be conducted to unswervingly compare the ability of each vaccine to prevent serious illness.

\section{Gaps in the literature}

Previous researches on the epidemiology of influenza transmission have failed to address the spread of the virus from one elderly to young health care worker during the aerosolgenerating process, especially in regards to other pathogens. This gap arises due to the fact that different pieces of literature fail to fully define the aerosol-generating process. There is a gap in the literature that fails to compare the ability of each vaccine to prevent serious illness caused by the influenza virus.

\section{Bibliography}

1. Atlanta: US Department of Health \& Human Services, Centers for Disease Control and a. Prevention.

2. Babazadeh, A., Afshar, Z.M., Javanian, M., Mohammadnia-Afrouzi, M., Karkhah, A., Masrour- 
a. Roudsari, J., Sabbagh, P., Koppolu, V., Vasigala, V.K. and Ebrahimpour, S., 2019.

b. Influenza vaccination and Guillain-Barré syndrome: Reality or fear. Journal of c. Translational Internal Medicine, 7(4), pp.137-142.

3. Barr, I.G. and Jelley, L.L., 2012. The coming era of quadrivalent human influenza a. vaccines. Drugs, 72(17), pp.2177-2185.

4. Centers for Disease Control and Prevention. (2013). Prevention and control of seasonal influenza

a. with vaccines: recommendations of the advisory committee on immunization practices -

b. United States, 2013-2014.

5. Chan, Y.W.D., Wong, M.L., Au, K.W. and Chuang, S.K., 2019. Seasonal influenza vaccine

a. effectiveness at primary care level, Hong Kong SAR, 2017/2018 winter. Human vaccines

b. \& immunotherapeutics, 15(1), pp.97-101.

6. Cunha, B. A.,2004. Influenza: historical aspects of epidemics and pandemics. Infectious a. Disease Clinics of North America, 18(1), 141-155.

7. Darvishian, M., van den Heuvel, E.R., Bissielo, A., Castilla, J., Cohen, C., Englund, H.,

a. Gefenaite, G., Huang, W.T., la Bastide-van Gemert, S., Martinez-Baz, I. and McAnerney,

b. J.M., 2017. Effectiveness of seasonal influenza vaccination in communitydwelling 
c. elderly people: an individual participant data meta-analysis of test-negative design case-

d. control studies. The Lancet Respiratory Medicine, 5(3), pp.200-211.

8. Fukushima, W. and Hirota, Y., 2017. Basic principles of test-negative design in evaluating

a. influenza vaccine effectiveness. Vaccine, 35(36), pp.4796-4800.

9. Girard, M.P., Tam, J.S., Pervikov, Y. and Katz, J.M., 2013. Report on the first WHO integrated

a. meeting on development and clinical trials of influenza vaccines that induce broadly

b. protective and long-lasting immune responses: Hong Kong SAR, China, 24-26 January

c. 2013. Vaccine, 31(37), pp.3766-3771.

10. Goodfellow, J.A. and Willison, H.J., 2016. Guillain-Barré syndrome: a century of

a. progress. Nature Reviews Neurology, 12(12), p.723.

11. Gupta, Y.K. \& Padhy, B.M., 2010. Issues in pharmacotherapy of 2009 H1N1 influenza

a. infection. Journal of Postgraduate Medicine, 56(4), 321-327.

12. Hamborsky, J. and Kroger, A. eds., 2015. Epidemiology and prevention of vaccinepreventable

a. diseases (pp. 279-96).

13. Horimoto, T. \& Kawaoka, Y. (2005). Influenza: lessons from past pandemics, warnings from 
a. current incidents. Nature Reviews Microbiology, 3, 591-600, doi:10.1038/nrmicro1208.

14. Jefferson, T., Rivetti, D., Rivetti, A., Rudin, M., Di Pietrantonj, C. and Demicheli, V., 2005.

a. Efficacy and effectiveness of influenza vaccines in elderly people: a systematic

b. review. The Lancet, 366(9492), pp.1165-1174.

15. Kavian, N., Hachim, A., Li, A.P., Cohen, C.A., Chin, A.W., Poon, L.L., Fang, V.J., Leung, N.H.,

a. Cowling, B.J. and Valkenburg, S.A., 2020. Assessment of enhanced influenza

b. vaccination finds that FluAd conveys an advantage in mice and older adults. Clinical \&

c. translational immunology, 9(2).

16. Kwong, E. W. Y., Pang, S. M. C., Choi, P. P., \& Wong, T. K. S., 2010. Influenzavaccine a. preference and uptake among older people in nine countries. Journal of Advanced b. Nursing, 66(10), 2297-2308.

17. Leung, J.C.K., 2007. Effectiveness of influenza vaccination among elderly home residents in

a. Hong Kong: a retrospective cohort study. Hong Kong Practitioner, 29(4), p.123.

18. Liu, W.M., van der Zeijst, B.A., Boog, C.J. \& Soethout, E.C. , 2011. Aging and impaired

a. immunity to influenza viruses: implications for vaccine development. Human Vaccine, 7 ,

b. 94-98. 
19. Mo, P.K.H. and Lau, J.T.F., 2015. Influenza vaccination uptake and associated factors among

a. elderly population in Hong Kong: the application of the Health Belief Model. Health

b. education research, 30(5), pp.706-718.

20. Mullooly, J.P., Bennett, M.D., Hornbrook, M.C., Barker, W.H., Williams, W.W., Patriarca, P.A.

a. \& Rhodes, P.H., 1994. Influenza vaccination programs for elderly persons: Cost-

b. effective in a health maintenance organization. Annuals of Internal Medicine, 121(12), 947-952.

21. Noda, T. and Kawaoka, Y., 2010. Structure of influenza virus ribonucleoprotein complexes and

a. their packaging into virions. Reviews in medical virology, 20(6), pp.380-391.

22. Praditsuwan, R., Assantachai, P., Wasi, C., Puthavatana, P. and Kositanont, U., 2005. The

a. efficacy and effectiveness of influenza vaccination among Thai elderly persons living in

b. the community. J Med Assoc Thai, 88(2), pp.256-64.

23. Reed, C., Kim, I.K., Singleton, J.A., Chaves, S.S., Flannery, B., Finelli, L., Fry, A., Burns, E.,

a. Gargiullo, P., Jernigan, D. and Cox, N., 2014. Estimated influenza illnesses and

b. hospitalizations averted by vaccination-United States, 2013-14 influenza

c. season. MMWR. Morbidity and mortality weekly report, 63(49), p.1151. 
24. Rondy, M., El Omeiri, N., Thompson, M.G., Levêque, A., Moren, A. and Sullivan, S.G., 2017.

a. Effectiveness of influenza vaccines in preventing severe influenza illness among adults:

b. A systematic review and meta-analysis of test-negative design case-control

c. studies. Journal of Infection, 75(5), pp.381-394.

25. Soema, P.C., Kompier, R., Amorij, J.P. and Kersten, G.F., 2015. Current and next generation

a. influenza vaccines: formulation and production strategies. European Journal of

b. Pharmaceutics and Biopharmaceutics, 94, pp.251-263.

26. Sung, L.C., Chen, C.I., Fang, Y.A., Lai, C.H., Hsu, Y.P., Cheng, T.H., Miser, J.S. and Liu, J.C.,

a. 2014. Influenza vaccination reduces hospitalization for acute coronary syndrome in

b. elderly patients with chronic obstructive pulmonary disease: a population-based cohort

c. $\quad$ study. Vaccine, 32(30), pp.3843-3849.

27. Verhees, R.A.F., Thijs, C., Ambergen, T., Dinant, G.J. and Knottnerus, J.A., 2019. Influenza

a. vaccination in the elderly: 25 years follow-up of a randomized controlled trial. No impact

b. on long-term mortality. PloS one, 14(5). 
28. Wong, C.M., Chan, K.P., Hedley, A.J. and Peiris, J.M., 2004. Influenza-associated mortality in

a. Hong Kong. Clinical Infectious Diseases, 39(11), pp.1611-1617.

29. World Health Organization. (2015). Influenza (seasonal) fact sheet No211.

30. Yu-lung, L.A.U., 2019. Scientific Committee on Vaccine Preventable Diseases (Doctoral a. dissertation, Faculty of Medicine, The University of Hong Kong).

31. Zhang, C.Q., Zhang, R., Chung, P.K., Duan, Y., fai Lau, J.T., Chan, D.K.C. and Hagger, M.S.,

a. 2018. Promoting influenza prevention for elderly people in Hong Kong using health

b. action process approach: study protocol. BMC public health, 18(1), pp.1-9. 